better communication among them established; generalities of findings magnified; additional benefits derived from comparing theories in diverse fields, explaining similarities and differences, and the validity and applicability of empirical work increased by planning individual studies as components of an explicit mosaic of research strategy."

The result of this wide plan has been, perhaps inevitably, that the papers tend to be mainly mathematical. This may be valuable since there is a limit to clinical research, and without the aid of other sciences psychology and psychiatry will reach an impasse. The papers here suggest that there is little danger of this. They range from "Biological and Cultural Evolution" to a "Critique of Stochastic Models for Learning". This journal will be a joy to the mathematician and no doubt will draw many into the study of behaviour who would otherwise treat it as too vague to be of interest.

Clifford Aluen

\section{THE NEED FOR OBSERVATIONS IN THE OLDER BRANCHES OF ASTRONOMY}

F

OR his presidential address last year to the Royal Astronomical Society and since published (Mon. Not. Roy. Astro. Soc., 115, 2 ; 1955), Dr. J. Jackson took as his subject "The Need for Observations in the Older Branches of Astronomy, especially Double Stars", and discussed three branches with which he has been associated for about forty years: double stars, stellar parallax and meridian astronomy. After the First World War Dr. Jackson did a considerable amount of work on double stars, collecting and discussing the observations made during the preceding thirty years. The observing programme at that time was largely restricted to the Struve stars, but now that many new double stars have been catalogued and that there has been a great decrease in the number of observers, it is necessary, said Dr. Jackson, to review the position. Sufficient knowledge regarding the average masses and distances of ordinary stars is now available to decide on those stars that are worth observing at intervals of twenty years and to distinguish them from those that cannot be expected to reveal orbital motion of more than a degree or two in a century.

The importance of this is shown by the following considerations regarding the $\Sigma$ stars. These were all picked up during 1824-27 with a $9 \frac{1}{2}$-in. refractor from among the hundred and twenty thousand brightest stars north of Dec. $-15^{\circ}$, and while the original list contained more than three thousand double stars, after various adjustments and rejections there remained 2,659 brighter than about magnitude 9 and with a separation less than 32". Before these were put on the Greenwich observing list, most of them had been observed at several epochs, and when Dr. Jackson discussed them in 1922 he found as follows: relative motion was shown by 649 , undoubted orbital motion by 449 , mere optical doubles in 161 cases, and in 39 pairs showing rather large relative motion it was difficult to decide whether they formed binary or optical systems; it is suggested that while the majority probably belonged to the latter class, some may have been true binaries with a rather large parallax which could be confirmed by trigonometrical observations. A very important point is that 2,010 of these stars showed no certain relative motion of the components, and the great majority of these must be real binaries with periods of many thousand years, as Dr. Jackson shows by an analysis of the figures. His conclusion is that the 2,010 stars which had shown no relative motion by 1920 , although true binaries, scarcely need any further attention until the end of this century. The 449 showing orbital motion, and also the 39 for which the nature of the motion is uncertain, should, he thinks, be observed at intervals of ten or twenty years until an orbit can be computed or the pair proved merely optical.

Turning to the $\mathrm{O \Sigma}$ stars, which were discovered with the 15-in. refractor at Pulkovo mostly during 184.-42, Dr. Jackson said that, owing to the closeness of the components and relatively greater difference in brightness than those just considered, they are more difficult to see and observe accurately. Hussey re-observed them with the 12-in. and 36-in. refractors at Lick Observatory, and when Dr. Jackson discussed them in 1923 he found that 135 showed orbital motion, 15 were optical systems, 5 showed motion of uncertain nature, and 270 showed no motion. In spite of the fact that these stars had been observed over a shorter interval of time than the $\Sigma$ stars and, in addition, accurate observation was more difficult, yot they contained a greater percentage of stars of importance for the future, a fact which is attributable to the stars being closer and the components brighter. Dr. Jackson's final remarks on the subject were: "I therefore conclude that one of the most urgent needs of observational astronomy at the present time is the systematic re-observation of important double stars for which we have already detected the beginnings of orbital motion. The position may not be quite so bad as it appears as there may be a number of measures of double stars not yet published, and the absence of a general catalogue giving observations later than 1927 makes it difficult to estimate the true position. Nevertheless the reports of the International Astronomical Union do indicate a steadily decreasing number of measures". (After preparing his address, Dr. Jackson became aware of the measures of many close double stars made by Dr. Muller, of Strasbourg, during a four months visit to Lick Observatory, and a footnote to the published address directs attention to this, with a short account of the first orbits calculated for thirteen double stars.)

The position with regard to parallax determinations is very satisfactory when compared with that about half a century ago. In Newcomb's book "The Stars", published in 1904, there is a list of seventytwo stars for which parallaxes were known ; of these, there were fifteen about which it was noted that the results were subject to more doubt than usual, and one was stated to be entirely unreliable. In contrast to this, the Yale Observatory published in 1953 a "General Catalogue of Trigonometric Stellar Parallaxes", which gives the parallaxes of 5,822 stars from about ten thousand determinations; this is the outcome of the great international scheme, devised about forty years ago by Schlesinger, for the mass determination of parallaxes, and was taken up at a dozen observatories which had long-focus telescopes. Several of the telescopes which have completed their programmes are at present used for the intensive observations of the nearest stars in the search for companions which are too close or too faint to be 
discovered except by the periodic displacement of the centre of light. A number of stars have been found to have companions which move in orbits with periods of a year or two. It is suggested that parallax observations for the next few years may be confined to filling in the gaps made by the omission of stars of early and late types, to the observation of certain specific stars, such as double stars or Vyssotsky's stars with dwarf characteristics, and to unravelling some of the larger discordances.

In the final part of his address, devoted to meridian astronomy, Dr. Jackson pointed out that at present the Royal Greenwich Observatory alone in Britain carries out observations with the meridian instrument for determining stellar positions. Several factors have contributed to this diminution of meridian observations in different countries, among which the economy of photography is important, and some may ask what justification there is for meridian astronomy. The answer is that, while we need accurate positions of only sufficient stars for reducing photographic plates, we require the proper motions of as many stars as possible, of every degree of brightness, of every spectral type, of every kind of variability, and in all galactic longitudes and latitude;, and all this involves the positions at two or more epochs. To determine the average distances of groups of stars too far away for trigonometrical calculations, proper motions are necessary, and they are thus important in investigations concerning the content of the Milky Way system at distances $\Theta x c e \theta d i n g$ a hundred parsecs, in addition to showing the systematic and random motions of the stars themselves. This led Dr. Jackson to a discussion of the fundamental systems used, of certain disadvantages inherent in some and also of various ideas for improvements. His address ended with a short account of a very important aspect of meridian astronomy-namely, accurate time determinationsin which the Royal Observatory plays a prominent part, and the results of the photographic zenith tube in action are awaited with interest.

\section{BRITISH JOURNAL OF RADIOLOGY}

\section{SIXTIETH ANNIVERSARY}

T HE May issue of the British Journal of Radiology is its diamond jubilee number. The Journal is the direct descendant of the Archives of Clinical Skiagraphy, which was the first journal to be devoted entirely to radiology. Its first number was dated April 2, 1896; but apparently it is not clear whether it was actually published then or in May of that year.

The British Journal of Radiology has had a great influence throughout the world in the development of medical radiology in its widest sense, and the jubilee number includes a number of historical reviews of particular interest. Prof. G. Stead deals with the contributions of physics to the progress of radiology during the past sixty years. It is perhaps singularly fortunate that many leading physicists have always devoted a great deal of effort to the advancement of the applications of physics to medicine. The first presidential address to the Roentgen Society, given by Silvanus 'Thompson, was apparently a remarkable beginning. Sir Oliver Lodge published a course of six lectures specially designed for medical prac- titioners. In the present number, Sir John Cockcroft writes on "The Future Role of Atomic Energy in Industry, Science and Medicine", Dr. Russell J. Reynolds reviews sixty years of radiology, and Dr. James T. Case summarizes the evolution of modern radiology. A point of historical interest is that in 1879 Sir William Crookes, without realizing it, had apparently obtained X-rays in the course of his experiments and had sent fogged photographic plates, which had been stored near his vacuum tubes, back to the manufacturers as defective. The account of X-ray apparatus by Mr. Cuthbert Andrews is illuminating. On the historical side, he mentions that "bound in the 1897 volume is what is undoubtedly the forerunner of our modern successful supplements -A Supplement to the Archives of the Röntgen Ray, entitled 'Radiography in Marine Zoology-'The British Echinodermata', by Dr. Wolfenden". Of particular interest to radiotherapists are the reviews by Dr. N. S. Finzi on radiotherapy and Dr. A. Lacassagne on radiobiology.

The development of British radiology has depended upon a number of factors. Among the most important of these are probably the recognition of the value of consultation in medicine with special reference to the many aspects of radiology, the applications of physics to both radiodiagnosis and radiotherapy and the interest throughout the years shown by many clinical radiologists in those aspects of fundamental science now called radiobiology. It is clear that the British Journal of Radiology has played a great part in facilitating these developments.

\section{ROCKEFELLER FOUNDATION GRANTS}

$\mathrm{T}$ H.E National Academy of Sciences, Washington, has initiated a study of the possible biological dangers to present and future generations of exposure to atomic radiation. 250,000 dollars has been awarded by the Rockefeller Foundation for this project. A biophysics research programme on the cultivation of individual animal cells has been devised by Dr. Theodore Puck and his associates in the University of Colorado, towards which the Foundation has made an award of 85,000 dollars. The Foundation has made a research grant of 60,000 dollars to Drs. J. J. Christensen and W. M. Myers for their work in the University of Minnesota in connexion with wheat stem rust. Research on the genetic factors of intelligence and emotional variation in dogs, directed by Dr. C. C. Little at the Roscoe B. Jackson Memorial Laboratory, Bar Harbor, Maine, receives a first-quarter grant of 50,000 dollars.

Dr. Edwin D. Kilbourne, of Cornell University Medical College, is working on latency or activation of viruses, towards the expenses of which the Foundation has granted 48,000 dollars. The University of Chicago is to receive 50,000 dollars for its programme of training in statistics for scientists. The State Institute for Human Genetics, Upsala, Sweden, has been awgrded 50,000 dollars. The work will be shared with the University of Upsala and the Royal Agricultural College. Prof. A. Rossi-Fanelli's work at the Institute of Biological Chemistry, University of Rome, will be supported over a period of four years by a grant of about 30,000 dollars, and that of Prof. P. Desnuelle at the Institute of Bio- 